\title{
EDITORIAL
}

\section{Minimizing Preventable Deaths: The Should Be Focus In Ethiopian Health System}

In the past two decades, the government of Ethiopia invested heavily on various sectors to bring holistic change. The health sector is one of the recipients of the investment with aim of minimizing preventable morbidities and mortalities, improving the health service coverage and betterment of health care delivery. Because of awareness creation works done and better access, the flow of patients to health care delivery units has increased significantly. As a result, tremendous changes are brought on almost all health indicators. Though access to health care gets better and referral system has somehow improved, advanced medical care delivery and specialized services are not yet well in place. Consequently, preventable hospital deaths are still occurring though health care providers work hard to provide better quality care to their patients with the available resources and training capacity.

Observations from day-to-day activity showed that most of these deaths are avoidable. Many patients each year suffer serious disability or death as a result of poor preparedness and facility. Some of the deaths are occurring due to delayed referral as a consequence of either poor diagnostic capacity at lower level or poor awareness of the seriousness of the condition by the family. Such cases, even if they are sent to the next level, they arrive too late giving no time for intervention at that level. Others die in hospital for several reasons, to mention some of them; lack of skilled human power, limited diagnostic facility, poor setups to treat critically ill patients and unavailability of the required medicines and supplies.

Given the considerable attention paid to health sector, implementers and policy makers need to consider the following improvement measures.

- Hospital leaders need to accurately identify the possible causes of death while patients are on care (type, phase of care). This should guide hospitals to implement a structured process for monthly mortality case reviews.

- The availability of ambulances in almost all districts is appreciable. However, proper communication system between the referring and recipient institution must be designed and implemented.

- Health professionals' clinical diagnostic and therapeutic capacity at all level should be boosted which must also be reinforced by appropriate diagnostic facility.

- Most critically ill patients die due to lack of basic and advanced care. Patients shouldn't die from lack of deserved care. To alleviate this, effort should be made to establish intensive care units at selected hospitals and avail necessary equipment, medicines and other supplies.

The current issue (Vol. 24, No 2), the second issue in the leap of EJHS to a quarterly publication, contains eight original articles on diverse issues, one review and four case reports focusing on various cases of rarity. I invite readers to read through these articles and appreciate or utilize the contents. I also encourage readers to forward comments and suggestions.

Abraham Haileamlak (MD)

Editor-in-Chief 